\title{
Desempenho zootécnico e custos de alimentação de juvenis de tainha submetidos à restrição alimentar
}

\author{
Eduardo Martins da Silva(1), Luís André Sampaio(2), Gabriel Bernardes Martins ${ }^{(2)}$, \\ Luis Alberto Romano(3) e Marcelo Borges Tesser ${ }^{(1)}$
}

\begin{abstract}
(1)Universidade Federal do Rio Grande (FURG), Instituto de Oceanografia (IO), Laboratório de Nutrição de Organismos Aquáticos, Rua do Hotel, no 2, CEP 96210-030 Rio Grande, RS. E-mail: edumsbio@yahoo.com.br, mbtesser@gmail.com (2)FURG, IO, Laboratório de Piscicultura Marinha e Estuarina. E-mail: sampaio@mikrus.com.br, martins.aqua@gmail.com ${ }^{(3)}$ FURG, IO, Laboratório de Patologia e Imunologia de Organismos Aquáticos. E-mail: dcluis@yahoo.com
\end{abstract}

Resumo - O objetivo deste trabalho foi avaliar o efeito de restrição alimentar sobre o desempenho zootécnico e custo de alimentação em juvenis de tainha (Mugil liza). Foram avaliados cinco taxas de alimentação 100, 80, 60, 40 e 20\% da saciedade, denominados F100\%, F $80 \%$, F60\%, F40\% e F20\% -, em triplicata. Juvenis com 0,21 $\pm 0,03 \mathrm{~g}(\mathrm{n}=30)$ foram distribuídos em 15 tanques de $200 \mathrm{~L}$. A estimativa do custo foi feita quanto à produção de mil juvenis de tainha. A sobrevivência foi superior a 93\%, e não houve influência dos tratamentos. A taxa de crescimento específico no tratamento $\mathrm{F} 100 \%$ foi de $5,96 \pm 0,18 \%$ por dia, e o peso final foi aproximadamente seis vezes maior que o inicial. Tainhas do tratamento F20\% apresentaram menor índice hepatossomático, hepatócitos e núcleos menores e menor reserva de glicogênio hepático em relação aos demais tratamentos. A redução da taxa de alimentação influenciou negativamente o crescimento de juvenis de tainha. No entanto, uma taxa de alimentação em torno de $72 \%$ da saciedade é capaz de promover o melhor aproveitamento do alimento consumido, com $28 \%$ de redução do custo da ração.

Termos para indexação: Mugil liza, conversão alimentar, custo de produção, saciedade.

\section{Growth performance and feeding costs of juvenile mullet subjected to feed restriction}

\begin{abstract}
The objective of this work was to evaluate the effect of restricted feeding on the growth and fed costs of juvenile mullet (Mugil liza). Five feeding levels - 100, 80, 60, 40 e 20\% satiation, named as F100\%, F $80 \%$, $\mathrm{F} 60 \%$, F $40 \%$ e F $20 \%$ - were evaluated in triplicate. Juvenile mullets with $0.21 \pm 0.03 \mathrm{~g}(\mathrm{n}=30)$ were randomly distributed in 15 tanks of $200 \mathrm{~L}$. Cost estimation was done for the production of one thousand juvenile mullets. Survival was higher than $93 \%$, and there was no influence of treatments. The specific growth rate of mullet in the $\mathrm{F} 100 \%$ treatment was $5.96 \pm 0.18 \%$ per day, and the final weight was six-fold higher than the initial one. Mullets of the F20\% treatment had the smallest liver somatic index, smallest hepatocytes and nuclei, and the smallest hepatic glycogen reserve, in comparison to fish of other treatments. The feeding level reduction hampered the juvenile mullet growth. However, a feeding level of about $72 \%$ satiation is capable of promoting a better utilization of the feed consumed, with $28 \%$ reduction of feed costs.
\end{abstract}

Index terms: Mugil liza, feed conversion, production cost, satiety.

\section{Introdução}

O manejo alimentar adequado é um dos fatores determinantes do sucesso do processo produtivo, uma vez que o consumo de alimentos influencia diretamente o crescimento e a conversão alimentar dos peixes (Mohseni et al., 2006). Restos alimentares decorrentes do manejo inadequado ou oriundos da baixa digestibilidade das rações podem comprometer a qualidade da água (Jana et al., 2006; Mohanta et al., 2008). As exigências nutricionais dos peixes também podem ser alteradas pelo consumo alimentar, pois a modificação da velocidade de passagem do bolo alimentar pelo estômago e intestino pode alterar a digestibilidade e absorção dos nutrientes (Fernández et al., 1998).

Algumas espécies têm maior crescimento e melhor conversão alimentar quando alimentadas até a saciedade, enquanto outras apresentam melhor desempenho quando alimentadas em níveis um pouco abaixo da saciedade. Neste caso, a melhor estratégia é alimentar os peixes não até a saciedade, e sim, em nível intermediário, para que se obtenha maior crescimento e melhor conversão alimentar (Sun et al., 2006). Assim, 
a obtenção de melhor conversão alimentar, com menor oferta de ração, é relevante, ao se considerar o elevado custo do alimento para a produção animal (Van Ham et al., 2003).

A tainha (Mugil liza) é uma espécie com hábito alimentar detritívoro (Vasconcelos Filho et al., 2009). É também euritérmica, com sobrevivência acima de $94 \%$, quando criada a temperaturas entre 20 e $30^{\circ} \mathrm{C}$ (Okamoto et al., 2006), e euri-halina, pois tolera desde água doce até água do mar (Sampaio et al., 2002). Essas características qualificam a espécie como apropriada para a aquicultura. No entanto, poucos são os trabalhos sobre o manejo alimentar e a nutrição de juvenis de tainha. Carvalho et al. (2010) determinaram que juvenis dessa espécie necessitam de $34 \%$ de proteína bruta em sua dieta.

O objetivo deste trabalho foi avaliar o efeito de diferentes níveis de restrição alimentar sobre o desempenho zootécnico e o custo de alimentação de juvenis de tainha.

\section{Material e Métodos}

Os juvenis de tainha foram capturados na praia do Cassino durante o período de verão. Foram transportados para o Laboratório de Piscicultura Estuarina e Marinha, do Instituto de Oceanografia da Fundação Universidade de Rio Grande (Furg), onde foram aclimatados ao cativeiro durante 30 dias e alimentados quatro vezes ao dia, até a saciedade, com uma dieta comercial INVE - NRD 5/8 (Inve Aquaculture Systems, Salt Lake City, UT, EUA) com $57 \%$ de proteína bruta, $14,5 \%$ de extrato etéreo e $19 \mathrm{MJ} \mathrm{kg}^{-1}$ de energia bruta, de acordo com os dados fornecidos pelo fabricante. A salinidade foi de $24 \%$, e a temperatura foi mantida em $28^{\circ} \mathrm{C}$. Diariamente, a água foi renovada e as fezes sifonadas.

Foram utilizados 450 peixes (peso médio de 0,21 $\pm 0,03 \mathrm{~g}, \mathrm{n}=30$ ), distribuídos aleatoriamente em 15 tanques de $200 \mathrm{~L}$ equipados com um sistema de filtro biológico individual e aeração constante. Os peixes foram submetidos a cinco taxas de alimentação, cada qual com três repetições: um grupo foi alimentado até a saciedade aparente (100\%) e, nos outros quatro grupos, os peixes foram alimentados com uma quantidade de ração equivalente a $80,60,40$ e $20 \%$ da saciedade, denominados $\mathrm{F} 100 \%$, F $80 \%$, F $60 \%$, F $40 \%$ e F $20 \%$. A saciedade aparente (100\%), em cada tanque, foi definida quando os peixes deixavam de buscar o alimento oferecido. Os peixes foram alimentados quatro vezes ao dia $(9,12,15$ e $18 \mathrm{~h})$ com a mesma dieta usada no período de aclimatação (INVE - NRD 5/8), e as quantidades de ração foram calculadas para cada período de alimentação, durante todo experimento, que teve duração de 30 dias.

Diariamente, foram determinadas: a temperatura; a concentração de oxigênio dissolvido, com um oxímetro digital YSI, modelo 55 Hexis, (YSI Incoporated, Yellow Springs, OH, EUA); a salinidade, por refratômetro óptico Atago, modelo 103 (Atago, Tóquio, Japão); a concentração de amônia total, pelo método de Solózano (1969); e a concentração de nitrito, de acordo com Bendschneider \& Robinson (1952). Duas vezes por semana, foi determinada a concentração de nitrato (Wood et al., 1967) e a alcalinidade (American Public Health Association, 2005). As leituras de transmitância foram feitas em espectrofotômetro digital Micronal B342 II (AJ Micronal, São Paulo, SP, Brasil), em $630 \mathrm{~nm}$ para amônia e $543 \mathrm{~nm}$ para nitrito e nitrato.

Os tanques foram sifonados diariamente para retirada das fezes, e a água perdida neste processo foi reposta imediatamente, em condições semelhantes de salinidade e temperatura. A temperatura foi controlada com aquecedores termostatizados, submersos, de $250 \mathrm{~W}$ Visi Therm (Aquaculture Systems, Mentor, OH, EUA). Aágua utilizada para as renovações foi estocada em tanques com aeração constante e aquecedores submersos que mantinham a temperatura em $28^{\circ} \mathrm{C}$. A salinidade de $24 \%$ foi obtida pela diluição da água marinha (35\%) com água doce, desclorada pela adição de tiossulfato de sódio $\left(7 \mathrm{mg} \mathrm{L}^{-1}\right)$.

Os peixes foram mantidos em jejum durante 24 horas, antes das biometrias, e foram anestesiados com benzocaína (50 $\left.\mathrm{mg} \mathrm{L}^{-1}\right)$ antes de serem pesados. A biometria inicial foi feita a partir de uma amostra de 30 peixes, antes de serem distribuídos nos tanques experimentais. Após 15 dias de experimento, 10 peixes de cada tanque foram medidos e, ao final (30 dias), todos os peixes foram pesados. Ao final do experimento, seis peixes de cada tratamento foram sacrificados por meio da exposição à concentração letal de benzocaína (100 $\mathrm{mg} \mathrm{L}^{-1}$ ), e seu fígado foi coletado. Os fígados foram pesados em balança analítica Sartorius MC1 AC210S (Sartorius, AG, Goettingen, Alemanha), com precisão de $0,1 \mathrm{mg}$, e imediatamente fixados em formol a $10 \%$ tamponado.

Pesq. agropec. bras., Brasília, v.48, n.8, p.906-912, ago. 2013 DOI: 10.1590/S0100-204X2013000800014 
Os fígados foram processados para análisehistológica padrão, com coloração de hematoxilina-eosina (HE) e também com o método PAS (ácido periódico de Schiff) sem hematoxilina. Foram examinados dois cortes de cada um dos seis fígados de cada tratamento. Para a morfometria hepática, foram medidas 300 células e seus respectivos núcleos em cada tratamento. Para a determinação da área coberta por glicogênio, foram contados os grânulos de glicogênio contidos em 10 campos por lâmina. As mensurações feitas para a morfometria foram realizadas em fotografias obtidas por câmera digital Olympus DP-72, acoplada em microscópio Olympus BX-51, com objetiva superplanocromática em aumento de 400 vezes.

A partir dos dados biométricos e morfométricos, foram calculados: a taxa de crescimento específico, $\mathrm{TCE}=[(\ln$ peso final - $\ln$ peso inicial $) /$ tempo $]$; o coeficiente de variação do peso e as mudanças no coeficiente de variação, $\Delta \mathrm{CV}=\mathrm{CV}$ final $/ \mathrm{CV}$ inicial; a conversão alimentar aparente, $\mathrm{CA}=$ consumo de alimento/ganho de biomassa; a taxa de eficiência proteica, TEP = ganho de peso/consumo de proteína; o índice hepatossomático, IHS = peso do fígado $\times 100$ peso corporal; o diâmetro médio dos hepatócitos, $\mathrm{DH}=$ (maior diâmetro celular + menor diâmetro celular)/2); o diâmetro médio dos núcleos dos hepatócitos, $\mathrm{DNH}=$ (maior diâmetro nuclear + menor diâmetro nuclear)/2; e a superfície ocupada pelo glicogênio por micrometro quadrado, $\mathrm{SOG}=(\sigma \mathrm{A} \times v \mathrm{~A}) / \mu$, em que: $\sigma \mathrm{A}$ é a constante, conforme a forma do grânulo, independentemente de seu tamanho; vA é o número contado; e $\mu$ é a área representada pelo reticulado da ocular. As medidas morfométricas dos hepatócitos foram realizadas de acordo com Romano et al. (1996).

O custo para alimentação dos juvenis de tainha foi estimado pela fórmula $\mathrm{C}_{1000}=\mathrm{CR} \times \mathrm{Cop} \times 1000$, em que: $\mathrm{C}_{1000}$ é o custo com alimentação para produzir 1.000 juvenis; CR é o custo da ração; e Cop é o consumo médio de alimento, em cada tanque, dividido pelo número de peixes presentes. A ração teve o custo de U\$21,60 $\mathrm{kg}^{-1}$.

Os resultados foram analisados quanto à homogeneidade e normalidade, pelos testes de Levene e Shapiro-Wilk, respectivamente. A análise de variância foi aplicada à todos os dados e, quando foi encontrada diferença significativa, o teste de Duncan foi utilizado. Curvas com os resultados de conversão alimentar e a taxa de eficiência proteica foram ajustadas a uma equação polinomial de segunda ordem $y=a x^{2}+b x+c$, em que x é a taxa de arraçoamento, c é a intersecção da curva, e a e b são coeficientes da regressão. A taxa de alimentação que resulta em máxima resposta $\left(\mathrm{T}_{\text {máx }}\right)$ é dada por $-\mathrm{b} / 2 \mathrm{a}$, em que o ponto de máxima resposta é o ponto a partir do qual não existe mais incremento no parâmetro analisado (Shearer, 2000). As análises estatísticas foram realizadas pelo programa Statistica 7.

\section{Resultados e Discussão}

Os valores de temperatura, salinidade, oxigênio dissolvido, concentração de amônia, nitrito, nitrato e alcalinidade não diferiram entre os tratamentos e tiveram valores de: $28,0 \pm 0,9{ }^{\circ} \mathrm{C}, 24,6 \pm 0,8 \%$, $6,24 \pm 0,29 \mathrm{mg} \mathrm{L}^{-1}, 0,13 \pm 0,09 \mathrm{mg} \mathrm{L} \mathrm{L}^{-1}$ de $\mathrm{N}_{-\mathrm{NH}_{3}}$, $0,33 \pm 0,24 \mathrm{mg} \mathrm{L}^{-1} \mathrm{de} \mathrm{N}-\mathrm{NO}_{2}, 1,0 \pm 0,31 \mathrm{mg} \mathrm{L}^{-1} \mathrm{de} \mathrm{N}-\mathrm{NO}_{3}$, e $131,25 \pm 5,30 \mathrm{mg} \mathrm{L}^{-1}$ de $\mathrm{CaCO}_{3}$, respectivamente.

$\mathrm{O}$ peso médio dos peixes (PM) diferiu significativamente entre todos os tratamentos, aos 15 dias e ao final do experimento (Figura 1). Aos 30 dias, o PM do tratamento $\mathrm{F} 100 \%$ foi seis vezes superior ao inicial. Os peixes do tratamento F20\% apenas dobraram o peso inicial. A redução da quantidade de alimento ofertado aos juvenis de tainha não influenciou a sobrevivência,

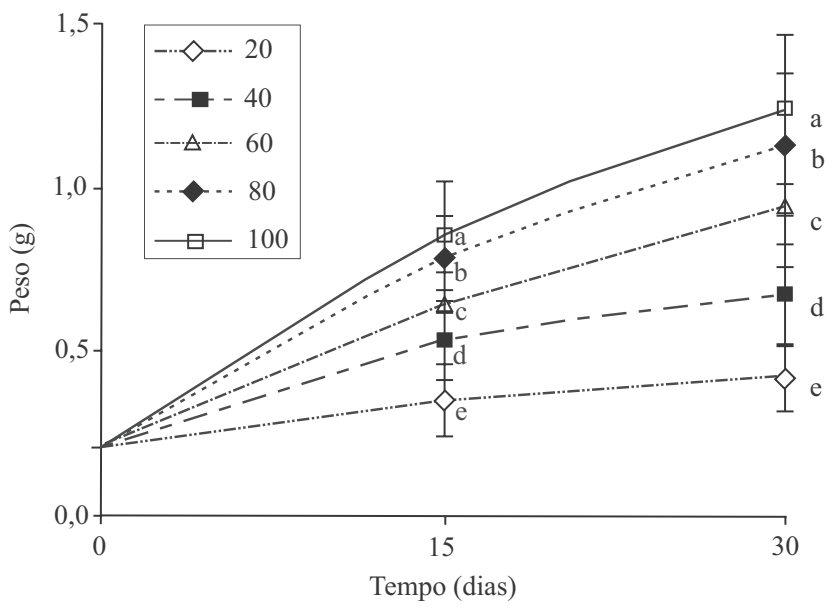

Figura 1. Peso médio de juvenis da tainha (Mugil liza) alimentados com dietas a diferentes taxas de arraçoamento, após 15 e 30 dias de experimento. Letras diferentes significam diferença estatística entre os tratamentos, pelo teste de Duncan, a 5\% de probabilidade. 
que foi superior a $93 \%$ e não apresentou diferença estatística entre os tratamentos (Tabela 1). A taxa de crescimento específico foi significativamente maior nos peixes do tratamento F100\%. Ahmed (2010) menciona que os peixes, de uma maneira geral, perdem peso quando a taxa de alimentação é menor do que a necessária para a manutenção diária do organismo; no entanto, com o aumento da disponibilidade de alimento e a consequente ingestão deste pelos peixes, ocorre um ajuste linear entre a taxa de crescimento específico e a taxa de alimentação. Esta relação entre o crescimento e a taxa de arraçoamento também foi observada nos estudos com juvenis de peixe-rei marinho (Odontesthes argentinensis) e para o Heteropneustes fossilis (Tesser \& Sampaio, 2006; Khan \& Adibi, 2010).

Não houve diferença significativa entre os tratamentos quanto ao coeficiente de variação do peso (Tabela 1). Van Ham et al. (2003) também observaram que juvenis de Scophtalmus maximus não apresentaram diferenças quanto ao coeficiente de variação de peso, quando alimentados até a saciedade ou com 65 e $35 \%$ da saciedade. A restrição alimentar pode causar o desenvolvimento de hierarquias, em que os peixes dominantes possuem maior acesso ao alimento, o que aumenta a variação de peso dentro de um grupo de peixes ao longo do tempo (Tucker et al., 2006). A uniformidade de tamanho é importante para a produção de juvenis, pois pode diminuir o canibalismo e a agressividade entre os peixes.

É importante determinar o nível de alimentação ótimo para os peixes, para que se tenha o melhor desempenho possível. Para isso, deve ser analisada a relação entre o crescimento do animal e seu consumode alimento, e deve ser calculada a conversão alimentar produzida pela interação destes fatores (Mohanta et al., 2008). Apesar de os peixes do grupo F20\% terem consumido a menor quantidade de alimento, eles apresentaram a menor conversão alimentar. Isso pode ser atribuído ao gasto energético para a manutenção do metabolismo, por ter sido utilizada a maior parte da energia ingerida e ter restado uma pequena parcela para o crescimento (Fang et al., 2010). Além disso, os peixes alimentados até a saciedade apresentaram menor conversão alimentar, em comparação aos peixes dos tratamentos $\mathrm{F} 80 \%$, F $60 \%$ e F40\%, como também observado por Sun et al. (2006). A redução da conversão alimentar denota um aproveitamento deficiente do alimento consumido, o que reduz a qualidade da água, pois aumenta a quantidade de resíduos liberados para o ambiente, além de aumentar o custo da produção aquícola (Seo et al., 2008).

A superfície ocupada por grânulos de glicogênio hepático dos peixes, em $\mathrm{F} 20 \%$, foi menor do que aquela encontrada nos hepatócitos dos peixes dos demais tratamentos $(\mathrm{p}<0,05)$ (Tabela 2). A síntese e a deposição de glicogênio e lipídios no fígado

Tabela 1. Índices zootécnicos (média \pm desvio-padrão) de juvenis da tainha, (Mugil liza) alimentados com dietas a diferentes níveis de arraçoamento ${ }^{(1)}$.

\begin{tabular}{lccccc}
\hline Variável (2) $^{(2)}$ & \multicolumn{4}{c}{ Taxas de alimentação (\% de saciedade aparente) } \\
\cline { 2 - 6 } & F20 & F40 & F60 & F80 & F100 \\
\hline Sobrevivência (\%) & $93,70 \pm 7,700$ & $96,50 \pm 3,400$ & $96,50 \pm 3,500$ & $98,80 \pm 2,100$ & $96,60 \pm 3,30$ \\
CV inicial (\%) & 16,89 & 16,89 & 16,89 & 16,89 & 16,89 \\
CV final (\%) & $22,98 \pm 1,490$ & $21,56 \pm 5,210$ & $19,78 \pm 4,390$ & $19,12 \pm 3,830$ & $17,55 \pm 3,30$ \\
CCV & $1,36 \pm 0,090$ & $1,27 \pm 0,310$ & $1,17 \pm 0,260$ & $1,13 \pm 0,230$ & $1,03 \pm 0,20$ \\
Peso inicial (g) & $0,21 \pm 0,030$ & $0,21 \pm 0,030$ & $0,21 \pm 0,030$ & $0,21 \pm 0,030$ & $0,21 \pm 0,03$ \\
Peso final (g) & $0,42 \pm 0,020 \mathrm{e}$ & $0,67 \pm 0,040 \mathrm{~d}$ & $0,94 \pm 0,040 \mathrm{c}$ & $1,13 \pm 0,010 \mathrm{~b}$ & $1,24 \pm 0,07 \mathrm{a}$ \\
TCE (\%) & $2,32 \pm 0,200 \mathrm{e}$ & $3,91 \pm 0,200 \mathrm{~d}$ & $5,03 \pm 0,150 \mathrm{c}$ & $5,64 \pm 0,030 \mathrm{~b}$ & $5,96 \pm 0,18 \mathrm{a}$ \\
CT (g) & $10,40 \pm 1,000 \mathrm{e}$ & $21,03 \pm 0,200 \mathrm{~d}$ & $31,70 \pm 0,100 \mathrm{c}$ & $42,60 \pm 0,160 \mathrm{~b}$ & $53,80 \pm 6,90 \mathrm{a}$ \\
CE (g) & $0,20 \pm 0,002 \mathrm{e}$ & $0,40 \pm 0,003 \mathrm{~d}$ & $0,60 \pm 0,002 \mathrm{c}$ & $0,81 \pm 0,003 \mathrm{~b}$ & $1,02 \pm 0,13 \mathrm{a}$ \\
CR (U\$) & $9,14 \pm 0,700 \mathrm{e}$ & $16,63 \pm 0,21 \mathrm{~d}$ & $24,81 \pm 0,970 \mathrm{c}$ & $34,13 \pm 0,130 \mathrm{~b}$ & $36,60 \pm 2,21 \mathrm{a}$ \\
\hline
\end{tabular}

(1) Médias seguidas de letras diferentes, nas linhas, diferem entre si pelo teste de Duncan, a 5\% de probabilidade. ${ }^{(2)} \mathrm{CV}$ inicial, coeficiente de variação do peso inicial; CV final, coeficiente de variação do peso final; $\Delta \mathrm{CV}$, mudanças no coeficiente de variação $(\Delta \mathrm{CV}=\mathrm{CV}$ final/CV inicial); TCE, taxa de crescimento específico; CT, consumo total de ração; $\mathrm{CE}$, consumo de energia (g); CR, custo da ração (cotação de $\mathrm{R} \$ 1,76$ ) para produção de mil juvenis. 
são influenciadas pelo consumo de energia (Evans et al., 2005). Assim, a menor quantidade de energia consumida pelos peixes do tratamento F20\% pode ter reduzido a atividade nuclear dos hepatócitos, o que resultou em menor quantidade de glicogênio estocado e menor diâmetro dessas células (Figuras 2 A e 2 B e Tabela 2). Medidas morfométricas no núcleo dos hepatócitos foram utilizadas por Carriquiriborde et al. (2007), para mostrar que o volume nuclear apresenta redução significativa decorrente da restrição alimentar. Outro indicador da condição nutricional de peixes é o índice hepatossomático (Everaarts et al., 1993). Este parâmetro não apresentou diferença entre os peixes dos tratamentos $\mathrm{F} 40 \%$ a $\mathrm{F} 100 \%$, o que corrobora os resultados de SOG encontrados no presente estudo. Independentemente do tratamento testado, não foram observadas alterações patológicas nos fígados dos peixes analisados no presente experimento.
O custo de alimentação para produção dos juvenis de tainha está intimamente relacionado à taxa de alimentação empregada, e o maior e o menor custos com a alimentação foram observados nos tratamentos F100\% e F20\%, respectivamente (Tabela 1). A análise de regressão polinomial indicou as taxas máximas de arraçoamento de 72,3 e $72,8 \%$ da saciedade, quando calculadas com a conversão alimentar (Figura 3 A) e a taxa de eficiência proteica (Figura $3 \mathrm{~B}$ ), respectivamente. Sun et al. (2006) já haviam recomendado $70 \%$ do arraçoamento máximo como o nível ideal de alimentação para juvenis de Rachycentron canadum. As taxas máximas de arraçoamento calculadas e o menor custo apresentado para o tratamento de $\mathrm{F} 80 \%$, comparado ao tratamento $\mathrm{F} 100 \%$, indicam que uma redução da quantidade de alimento ofertado melhora o aproveitamento dos nutrientes, além de reduzir em $28 \%$ os custos com ração.

Tabela 2. Análise morfométrica (média \pm desvio padrão) realizada em hepatócitos de juvenis da tainha (Mugil liza) alimentados com dietas a diferentes taxas de arraçoamento $^{(1)}$.

\begin{tabular}{lccccc}
\hline Variáveis $^{(2)}$ & \multicolumn{5}{c}{ Taxas de alimentação (\% de saciedade aparente) } \\
\cline { 2 - 6 } & F20 & F40 & F60 & F80 & F100 \\
\hline SOG $\left(\mu \mathrm{m}^{-2}\right)$ & $143,1 \pm 44,20 \mathrm{~b}$ & $241,5 \pm 10,3 \mathrm{a}$ & $273,2 \pm 22,40 \mathrm{a}$ & $263,4 \pm 29,10 \mathrm{a}$ & $220,4 \pm 32,60 \mathrm{a}$ \\
$\mathrm{DNH}(\mu \mathrm{m})$ & $3,1 \pm 0,04 \mathrm{c}$ & $3,3 \pm 0,3 \mathrm{bc}$ & $4,0 \pm 0,20 \mathrm{a}$ & $3,7 \pm 0,20 \mathrm{ab}$ & $3,7 \pm 0,40 \mathrm{ab}$ \\
$\mathrm{DH}(\mu \mathrm{m})$ & $9,1 \pm 0,20 \mathrm{c}$ & $10,5 \pm 1,1 \mathrm{bc}$ & $14,9 \pm 2,00 \mathrm{a}$ & $12,8 \pm 1,20 \mathrm{ab}$ & $13,9 \pm 2,30 \mathrm{a}$ \\
IHS $(\%)$ & $1,8 \pm 0,43 \mathrm{~b}$ & $2,2 \pm 0,4 \mathrm{ab}$ & $2,5 \pm 0,71 \mathrm{a}$ & $2,71 \pm 0,51 \mathrm{a}$ & $2,82 \pm 0,46 \mathrm{a}$ \\
\hline
\end{tabular}

${ }^{(1)}$ Médias seguidas de letras diferentes, nas linhas, diferem entre si, pelo teste de Duncan, a $5 \%$ de probabilidade. ${ }^{(2)}$ SOG, superfície ocupada por glicogênio; DNH, diâmetro médio do núcleo dos hepatócitos; DH, diâmetro médio dos hepatócitos; IHS, índice hepatossomático.
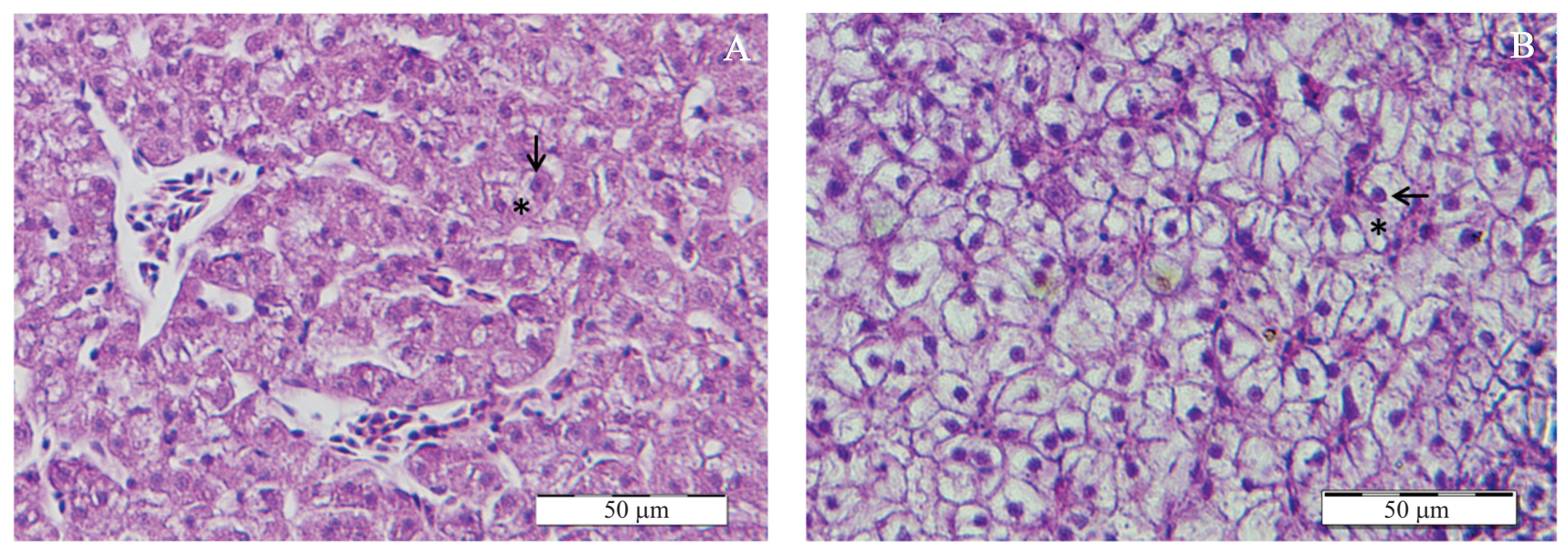

Figura 2. Fotomicrografias de cortes histológicos de fígados de juvenis de tainha (Mugil liza) alimentados com 20\% (A) e $100 \%$ da saciedade (B). Cortes corados em hematoxilina e eosina. Aumento de 400 vezes. *Citoplasma. $\leftarrow \downarrow$ Núcleo. 

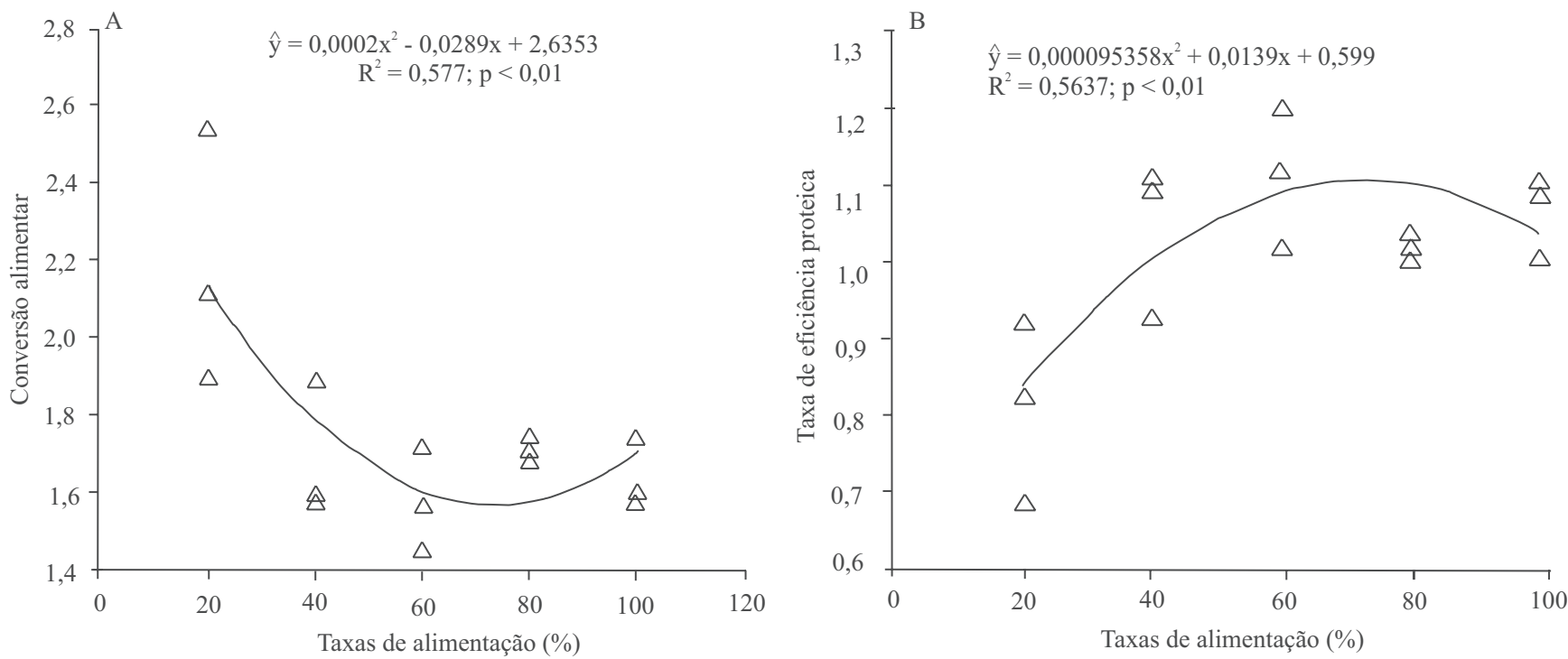

Figura 3. Regressões polinomiais calculadas de conversão alimentar aparente (A) e taxa de eficiência proteica (B) de juvenis de tainha, (Mugil liza) alimentados com dietas a diferentes taxas de arraçoamento. A taxa máxima ( $\mathrm{T}_{\text {máx. }}$.) de alimentação que promove a melhor conversão alimentar e a taxa de eficiência proteica foram calculadas a partir da equação de regressão quadrática $-\mathrm{b} / 2 \mathrm{a}$.

\section{Conclusão}

Taxas de alimentação com $72 \%$ da saciedade promovem desempenho zootécnico satisfatório, com menor investimento em ração, em juvenis de tainha.

\section{Agradecimentos}

Ao Conselho Nacional de Desenvolvimento Científico e Tecnológico (CNPq), pela concessão de bolsas.

\section{Referências}

AHMED, I. Response to the ration levels on growth, body composition, energy, and protein maintenance requirement of the Indian catfish (Heteropneustes fossilis - Bloch 1974). Fish Physiology Biochemistry, v.36, p.1133-1143, 2010. DOI: 10.1007/ s10695-010-9391-X.

AMERICAN PUBLIC HEALTH ASSOCIATION. Standard methods for the examination of water and wastewater. $21^{\text {th }}$ ed. Washington: APHA, 2005.

BENDSCHNEIDER, K.; ROBINSON, R.J. A new spectrophotometric method for the determination of nitrite in sea water. Journal of Marine Research, v.11, p.87-96, 1952.

CARRIQUIRIBORDE, P.; DE LUCA, J.C.; DULOUT, F.N.; RONCO, A.E. Nucleolar variation in response to nutritional condition in juvenile pejerrey Odontesthes bonariensis
(Valenciennes). Journal of Fish Biology, v.70, p.947-958, 2007. DOI: $10.1111 /$ j.1095-8649.2007.01357.x.

CARVALHO, C.V.A. de; BIANCHINI, A.; TESSER, M.B.; SAMPAIO, L.A. The effect of protein levels on growth, postprandial excretion and tryptic activity of juvenile mullet Mugil platanus (Günther). Aquaculture Research, v.41, p.511-518, 2010. DOI: 10.1111/j.1365-2109.2009.02340.x.

EVANS, J.J.; PASNIK, D.J.; PERES, H.; LIM, C.; KLESIUS, P.H. No apparent differences in intestinal histology of channel catfish (Ictalurus punctatus) fed heat-treated and non-heat-treated raw soybean meal. Aquaculture Nutrition, v.11, p.123-129, 2005. DOI: $10.1111 /$ j.1365-2095.2004.00329.x.

EVERAARTS, J.M.; SHUGART, L.P.; GUSTIN, M.K.; HAWKINGS, W.E.; WALKER, W.W. Biological markers in fish: DNA integrity, hematological parameters and liver somatic index. Marine Environmental Research, v.35, p.101-107, 1993. DOI: 10.1016/0141-1136(93)90021-Q.

FANG, J.; TIAN, X.; DONG, S. The influence of water temperature and ration on the growth, body composition and energy budget of tongue sole (Cynoglossus semilaevis). Aquaculture, v.299, p.106-114, 2010. DOI: 10.1016/j.aquaculture.2009.11.026.

FERNÁNDEZ, F.; MIGUEL, A.G.; GUINEA, J.; MARTINEZ, R. Digestion and digestibility in gilthead sea bream (Sparus auratus): the effect of diet composition and ration size. Aquaculture, v.166, p.67-84, 1998. DOI: 10.1016/S0044-8486(98)00272-5.

JANA, S.N.; GARG, S.K.; BARMAN, U.K.; ARASU, A.R.T.; PATRA, B.C. Effect of varying dietary protein levels on growth and production of Chanos chanos (Forsskål) in inland saline groundwater: laboratory and field studies. Aquaculture 
International, v.14, p.479-498, 2006. DOI: 10.1007/ s10499-006-9050-5.

KHAN, M.A.; ABIDI, S.F. Optimum ration level for better growth, conversion efficiencies and body composition of fingerling Heteropneustes fossilis (Bloch). Aquaculture International, v.18, p.175-188, 2010. DOI: 10.1007/s10499-008-9234-2.

MOHANTA, K.N.; MOHANTY, S.N.; JENA, J.K.; SAHU, N.P. Protein requirement of silver barb, Puntius gonionotus fingerlings. Aquaculture Nutrition, v.14, p.143-152, 2008. DOI: 10.1111/j.13 65-2095.2007.00514.x.

MOHSENI, M.; POURKAZEMI, M.; BAHMANI, M.; FALAHATKAR, B.; POURALI, H.R.; SALEHPOUR, M. Effects of feeding rate and frequency on growth performance of yearling great sturgeon, Huso huso. Journal of Applied Ichthyology, v.22, p.278-282, 2006. DOI: 10.1111/j.1439-0426.2007.00968.x.

OKAMOTO, M.H.; SAMPAIO, L.A.N. de; MAÇADA, A. de P. Efeito da temperatura sobre o crescimento e a sobrevivência de juvenis de tainha Mugil platanus Günther, 1880. Atlântica, v.28, p.61-66, 2006.

ROMANO, L.A.; FERDER, M.D.; STELLA, I.Y.; INSERRA, F.; FERDER, L.F. High correlation in renal tissue between computed image analysis and classical morphometric analysis. The Journal of Histotechnology, v.19, p.121-123, 1996. DOI: $10.1179 / 014788896794730810$.

SAMPAIO, L.A.; WASIELESKY, W.B.; MIRANDA FILHO, K.C. Effect of salinity on acute toxicity of ammonia and nitrite to juvenile Mugil platanus. Bulletin of Environmental Contaminant and Toxicology, v.68, p.668-674, 2002. DOI: 10.1007/s001280306.

SEO, J.-Y.; LEE, S.-M. Effects of dietary macronutrient level and feeding frequency on growth and body composition of juvenile rockfish (Sebastes schlegelii). Aquaculture International, v.16, p.551-560, 2008. DOI: 10.1007/s10499-008-9165-y.

SHEARER, K.D. Experimental design, statistical analysis and modelling of dietary nutrient requirement studies for fish: a critical review. Aquaculture Nutrition, v.6, p.91-102, 2000. DOI: 10.1046/j.1365-2095.2000.00134.x.

SOLÓRZANO, L. Determination of ammonia in natural waters by the phenol hypochlorite method. Limnology and Oceanography, v.14, p.799-801, 1969. DOI: 10.4319/lo.1969.14.5.0799.

SUN, L.H.; CHEN, H.R.; HUANG, L.M.; WANG, Z.; YAN, Y. Growth and energy budget of juvenile cobia (Rachycentron canadum) relative to ration. Aquaculture, v.257, p.214-220, 2006. DOI: 10.1016/j.aquaculture.2006.02.074.

TESSER, M.B.; SAMPAIO, L.A. Criação de juvenis de peixe-rei (Odontesthes argentinensis) em diferentes taxas de arraçoamento. Ciência Rural, v.36, p.1278-1282, 2006. DOI: 10.1590/ S0103-84782006000400036.

TUCKER, B.J.; BOOTH, M.A.; ALLAN, G.L.; BOOTH, D.; FIELDER, D.S. Effects of photoperiod and feeding frequency on performance of newly weaned Australian snapper Pagrus auratus. Aquaculture, v.258, p.514-520, 2006. DOI: 10.1016/j. aquaculture.2006.03.033.

VAN HAM, E.H.; BERNTSSEN, M.H.G.; IMSLAND, A.K.; PARPAURA, A.C.; WENDELAAR BONGA, S.E.; STEFANSSON, S.O. The influence of temperature and ration on growth, feed conversion, body composition and nutrient retention of juvenile turbot (Scophtalmus maximus). Aquaculture, v.217, p.547-558, 2003. DOI: 10.1016/S0044-8486(02)00411-8.

VASCONCELOS FILHO, A. de L.; NEUMANN-LEITÃO, S.; ESKINAZI-LEÇA, E.; OLIVEIRA A.M.E. de; PORTO-NETO, F. de F. Hábitos alimentares de consumidores primários da ictiofauna do sistema estuarino de Itamaracá (Pernambuco - Brasil). Revista Brasileira de Engenharia de Pesca, v.4, p.21-31, 2009.

WOOD, E.D.; ARMSTRONG, F.A.J.; RICHARDS, F.A. Determination of nitrate in sea water by cadmium-copper reduction to nitrite. Journal of the Marine Biological Association of the United Kingdom, v.47, p.23-31, 1967. DOI: 10.1017/ S002531540003352X.

Recebido em 17 de maio de 2011 e aprovado em 27 de fevereiro de 2013 